\title{
Research on Adaptive UKF Algorithm in Integrated Navigation System
}

\author{
Jianjun $\mathrm{Li}^{1}$, Junshan $\mathrm{Li}^{1}$ and Jianye Yang ${ }^{2}$ \\ ${ }^{1}$ The institute of information science \& technology Guangdong University of Foreign Studies South China Business College \\ Guangzhou, China \\ ${ }^{2}$ Rocket Sergeant school Qingzhou, China
}

\begin{abstract}
According to the characteristics of the combined navigation system and the adaptive UKF filtering algorithm, the navigation accuracy of the integrated navigation system is improved. Firstly, the algorithm of the UKF filtering algorithm is analyzed and the characteristics of the UKF algorithm are summarized, and the adaptive factor is introduced into the UKF filtering algorithm by the adaptive estimation principle, and the improved adaptive UKF algorithm is obtained, and the algorithm is applied to the integrated navigation system. Finally, the simulation calculation of the computer is carried out. The simulation results of adaptive UKF algorithm applied to integrated navigation system are improved, and the results are compared with the results of UKF algorithm. The experimental results show that the improved adaptive UKF can effectively suppress the influence of the initial value selection of the integrated navigation system, reduce the disturbance of the system state model, and effectively improve the precision of the integrated navigation.
\end{abstract}

Keywords-component; adaptive estimation principle; Unscented Kalman Filter; integrated navigation

\section{INTRODUCTION}

At present, the navigation systems commonly used in navigation guidance are satellite navigation system (GNSS) and strapdown inertial navigation system (SINS). These two kinds of navigation systems have their own advantages and disadvantages. The GNSS system has high positioning and speed measurement accuracy and no accumulation of error, but the output information is discontinuous and easy to be disturbed; SINS is developed on the basis of the platform inertial navigation system, which has the advantages of high reliability, strong autonomy, high short-time precision and continuous output, but its error accumulates with time. By combining the two components, we can achieve complementary advantages and improve the navigation accuracy and comprehensive performance of the navigation system. At present, this integrated navigation system is widely used in aerospace, aerospace and other fields such as space vehicle, launch vehicle, various aircraft, tactical missile and so on. It is an ideal integrated navigation system ${ }^{[1-4]}$.

The extended kalman filter (EKF) is widely used in the integrated navigation system, and the auxiliary information is used as the view measurement, and the integrated navigation system is most estimated, and the high precision navigation signal can be obtained. The basic idea is to linearize the nonlinear functions, to carry out the first order Taylor expansion of the nonlinear system model, and then use the Kalman algorithm to calculate. The disadvantage of EKF is that the linearization of the system model will introduce a larger truncation error, the filtering performance decreases or even diverges, and the calculation of the Jacobian matrix is complex and large, so the robustness of the EKF is poor. Based on the extended kalman filter algorithm, the knscented kalman filter (UKF) is proposed. The idea of UKF is to approximate the posterior probability density function of the state of the nonlinear system by UT transformation. By designing a small number of Sigma points, the one or two order statistical properties of the random direction are calculated. The algorithm is widely used in robot positioning and integrated navigation system because of its small computation and high accuracy. Although UKF can overcome some problems of EKF, in practical application, UKF has a large amount of computation and not high real-time, and the noise has the statistical characteristics of Gauss distribution, which is sensitive to the value of initial values, poor robustness and poor tracking ability. In view of the problem of the existence of UKF and combining the adaptive estimation theory, an improved UKF algorithm is proposed in this paper. The algorithm improves the real-time performance of the navigation calculation, effectively inhibits the influence of the deviation of the initial value selection of the integrated navigation system, reduces the influence of the disturbance of the system state model, and improves the precision of the integrated navigation.

\section{UNSCENTED KALMAN FILTER}

A discrete nonlinear system is set up.

$$
\begin{gathered}
x_{k+1}=f\left(x_{k}, u_{k}, k\right)+\omega_{k} \\
y_{k}=h\left(x_{k}, u_{k}, k\right)+v_{k}
\end{gathered}
$$

In the formula, $x_{\bar{H}}$ is the system state vector, $u_{\bar{R}}$ is the input control vector, $\omega_{k}$ is the system noise vector, $y_{k}$ is the observation vector, and $v_{k i}$ is the measurement noise vector.

A series of sampling points are selected near $\hat{f}_{k}$. The mean and covariance of these sampling points are $\hat{x}_{\hat{k}}$ and $P_{k}$ respectively. A series of sampling points are selected near $R(k \| k)$. The mean and covariance of these sampling points are 
$f(k \mid k)$ and $P^{\prime}(k \mid k)$ respectively. These sampling points pass through the nonlinear system and produce the corresponding transformation sampling points, and the calculated mean and covariance can be obtained by calculating the sampling points.

The steps of the UKF filtering algorithm ${ }^{[5-7]}$ are as follows:

(1)Initialization

$$
\hat{x}_{0}=E\left[x_{0}\right], \quad P_{0}=E\left[\left(x_{0}-\hat{x}_{0}\right)\left(x_{0}-\hat{x}_{0}\right)^{\mathrm{T}}\right] \quad k \geq 1
$$

(2)Calculating sampling point

$$
\chi_{k-1}=\left[\hat{x}_{k-1} \hat{x}_{k-1}+\sqrt{n+\tau}\left(\sqrt{P_{k-1}}\right)_{i} \hat{x}_{k-1}-\sqrt{n+\tau}\left(\sqrt{P_{k-1}}\right)_{i}\right] i=1,2 \cdots, n
$$

(3)Time update

$$
\begin{gathered}
\chi_{k \mid k-1}=f\left(\chi_{k-1}, u_{k-1}, k-1\right) \\
\hat{x}_{\bar{k}}=\sum_{i=0}^{2 n} W_{i} \chi_{i, k \mid k-1} \\
P_{\bar{k}}=\sum W_{i}\left[\chi_{i, k \mid k-1}-\hat{x}_{\bar{k}}\right]\left[\chi_{i, k \mid k-1}-\hat{\chi}_{\bar{k}}\right]^{\mathrm{T}}+Q_{k}
\end{gathered}
$$

Among them, $Q_{k}$ is the noise covariance of the system.

$$
\begin{gathered}
y_{k \mid k-1}=h\left(\chi_{k \mid k-1}, u_{k}, k\right) \\
\hat{y}_{\bar{k}}=\sum_{i=0}^{2 n} W_{i} y_{i, k \mid k-1}
\end{gathered}
$$

(4)Measurement update

$$
P_{\tilde{y}_{k} \tilde{y}_{k}}=\sum_{i=0}^{2 n} W_{i}\left[y_{i, k \mid k-1}-\hat{y}_{\bar{k}}\right]\left[y_{i, k \mid k-1}-\hat{y}_{\bar{k}}\right]^{\mathrm{T}}+R_{k}
$$

Among them, $\mathbb{R}_{k}$ is a measurement of noise covariance.

$$
\begin{gathered}
P_{x_{k} y_{k}}=\sum_{i=0}^{2 n} W_{i}\left[\chi_{i, k \mid k-1}-\hat{x}_{\bar{k}}\right]\left[y_{i, k \mid k-1}-\hat{y}_{\bar{k}}\right]^{\mathrm{T}} \\
K_{k}=P_{x_{k} y_{k}} P_{\hat{y}_{k} \hat{y}_{k}}^{-1}
\end{gathered}
$$

$$
\begin{gathered}
\hat{x}_{k}=\hat{x}_{\bar{k}}+K_{k}\left(y_{k}-\hat{y}_{\bar{k}}\right) \\
P_{k}=P_{\bar{k}}-K_{k} P_{\hat{y}_{k} \hat{y}_{k}} K_{k}^{\mathrm{T}}
\end{gathered}
$$

Prediction error vector $Y=y_{k}-\hat{y}_{\bar{k}}$.

According to the principle of UKF algorithm, the basic idea of the UKF algorithm for system state estimation is consistent with the linear Kalman filter. It is based on the state one step prediction to add a measurement related adjustment correction, which mainly has the following characteristics:

(1) UKF does not need to linearize the system equation and the measurement equation, that is, it does not need to solve the nonlinear model of the system, avoids the calculation of the Jacobi matrix, and the approximation of the nonlinear object can reach the approximation of the two order, and the UKF can adapt to the estimation under the strong nonlinear condition.

(2) The UKF algorithm is applicable to any nonlinear model, and its implementation is simple and the accuracy is higher than EKF.

\section{IMPROVED ADAPTIVE UKF}

Through the analysis above, UKF has many advantages different from the traditional Kalman filter and EKF filter, but there are some shortcomings that affect the filtering precision in the actual application process. With the increase of the dimension of the system, the distance between the Sigma set and the mean point increases with the increase of the dimension of the system. Although the mean and variance of the random variable can be maintained, it is not a local sample, but the filtering error of the system is increased. In addition, the UKF is more sensitive to the initial value of the filter. In the UKF filtering algorithm, the selection of the initial value of the filter is passed through the equation through the equation. The accuracy of the observation is directly affected by the delivery. Aiming at some problems of UKF, combining with the principle of adaptive estimation, the adjustment error of UKF algorithm in integrated navigation system is adjusted by using the observation information to improve the precision of the integrated navigation system.

In the process of UKF filtering algorithm, the principle of variance transfer is adopted to reduce the influence of the initial value of the filter on the state equation and the observation equation, and to adjust its application in the filtering process ${ }^{[5]}$. The adaptive factor $\alpha_{k}\left(0<\alpha_{k} \leq 1\right)$ is chosen to rewrite equation (10) (11) (14) as follows:

$$
\begin{gathered}
\bar{P}_{\tilde{y}_{k} \tilde{y}_{k}}=\frac{1}{\alpha_{k}} \sum_{i=0}^{2 n} W_{i}\left[y_{i, k \mid k-1}-\hat{y}_{\bar{k}}\right]\left[y_{i, k \mid k-1}-\hat{y}_{\bar{k}}\right]^{\mathrm{T}}+R_{k} \\
\bar{P}_{x_{k} y_{k}}=\frac{1}{\alpha_{k}} \sum_{i=0}^{2 n} W_{i}\left[\chi_{i, k \mid k-1}-\hat{x}_{\bar{k}}\right]\left[y_{i, k \mid k-1}-\hat{y}_{\bar{k}}\right]^{\mathrm{T}}
\end{gathered}
$$




$$
\bar{P}_{k}=\frac{1}{\alpha_{k}} P_{\bar{k}}-K_{k} P_{\hat{y}_{k} \hat{y}_{k}} K_{k}^{\mathrm{T}}
$$

$\alpha_{k}$ can not only balance the weight of prediction information and observation information adaptively, but also adjust the influence of system model disturbance on filtering. The value of $\alpha_{k}$ is:

$$
\alpha_{k}=\left\{\begin{array}{cc}
1 & \operatorname{tr}\left(Y Y^{T}\right) \leq \operatorname{tr}\left(P_{\tilde{y}_{k} \tilde{y}_{k}}\right) \\
\frac{\operatorname{tr}\left(P_{\tilde{y}_{k} \tilde{y}_{k}}\right)}{\operatorname{tr}\left(Y Y^{T}\right)} & \operatorname{tr}\left(Y Y^{T}\right)>\operatorname{tr}\left(P_{\tilde{y}_{k} \tilde{y}_{k}}\right)
\end{array}\right.
$$

According to formula (18), $\alpha_{k}$ can improve the UKF algorithm. When the filtering initial value is selected and the system state model is disturbed, $\alpha_{k}<1$. Its meaning indicates that the predictive information of the mathematical model of the system should be as small as possible for the filtering solution. When the system state model appears the difference Chang Bo movement, the ${ }^{\alpha_{k}}$ approximation is 0 . From the above analysis, we can see that ${ }^{\alpha_{k}}$ can adjust the influence of various state variables on integrated navigation system adaptively with covariance observations.

\section{SINS/GPS INTEGRATED NAVIGATION MATHEMATICAL MODELE}

Taking the SINS/GPS integrated navigation system as the research object, the system adopts the position and speed information synthesis method in the loose combination. The difference of the position and speed information of the GPS and SINS output is taken as the measurement value. After the state error is estimated by the Calman filter, the navigation parameters are corrected by the output correction method.

Error state equation of integrated navigation system

$$
\dot{X}(t)=F(t) X(t)+G(t) W(t)
$$

Among them, $X(t)$ is a system state variable, which consists of 15 states according to the SINS and GPS system error models; $W(t)$ is the system noise matrix, $F(t)$ and $G(t)$ are coefficient matrices respectively, and the parameters of each parameter refer to the literature ${ }^{[8-10]}$.

\section{Measurement equation of integrated navigation system}

The combination of speed and position is selected as the measurement. The specific scheme is to use the speed information of the East, north, the sky direction of the SINS system and the corresponding velocity information of the corresponding GPS in these three directions as the velocity measurement information. The difference between the latitude and latitude and the height information of the SINS system and the corresponding position information of the corresponding GPS is used as the location measurement information. Combining velocity measurement information with position measurement information is the measurement equation of integrated navigation system.

The position measurement equation is as follows:

$$
Z_{P}(t)=\left[\begin{array}{c}
L_{I}-L_{G} \\
\lambda_{I}-\lambda_{G} \\
h_{I}-h_{G}
\end{array}\right]=H_{p}(t) X(t)+V_{P}(t)
$$

In the formula, $H_{P}=\left[\begin{array}{lllll}0_{3 \times 6} & \operatorname{diag}\left\{\begin{array}{llll}R & R \cos L & 1\end{array}\right\} & 0_{3 \times 9}\end{array}\right]$

Velocity measurement equation

$$
Z_{V}(t)=\left[\begin{array}{c}
V_{I N}-V_{G N} \\
V_{I E}-V_{G E} \\
V_{I U}-V_{G U}
\end{array}\right]=H_{V}(t) X(t)+V_{V}(t)
$$

In the formula, $H_{V}=\left[\begin{array}{lllll}0_{3 \times 3} & \operatorname{diag}\left\{\begin{array}{llll}1 & 1 & 1\end{array}\right\} 0_{3 \times 12}\end{array}\right]$

The equations of measurement of GPS and SINS system and velocity measurement equations are combined to get the measurement equation of the system.

$$
Z(t)=\left[\begin{array}{c}
Z_{P}(t) \\
\vdots \\
Z_{V}(t)
\end{array}\right]=\left[\begin{array}{c}
H_{P}(t) \\
\vdots \\
H_{V}(t)
\end{array}\right] X(t)+\left[\begin{array}{c}
V_{P}(t) \\
\vdots \\
V_{V}(t)
\end{array}\right]=H(t) X(t)+V(t)
$$

\section{SimULATION AND ANALYSIS}

Taking the SINS/GPS integrated navigation system of flight carrier as an example, the system uses the northeastern sky navigation coordinate system. The state variables of the system include geographical longitude error, latitude error, height error, eastward velocity error, north direction velocity error, sky velocity error, gyro drift error, accelerometer error and attitude angle error.

$$
X=\left[\begin{array}{lllllllllllllll}
\Phi_{e} & \Phi_{n} & \Phi_{u} & \delta V_{e} & \delta V_{n} & \delta V_{u} & \delta L & \delta \lambda & \delta h & \varepsilon_{x} & \varepsilon_{y} & \varepsilon_{z} & \Delta_{x} & \Delta_{y} & \Delta_{z}
\end{array}\right]^{T}
$$

The measured state variables of the system include latitude, longitude and height of the carrier, eastward velocity, northward velocity and direction velocity.

$$
Z=\left[\begin{array}{llllll}
\delta \phi & \delta \lambda & \delta h & \delta V_{e} & \delta V_{n} & \delta V_{u}
\end{array}\right]^{T}
$$

It is assumed that the error characteristics of the gyroscope and the accelerometer are the same, the initial horizontal error angle is 0.1 degrees, the initial azimuth error angle is 0.5 degrees, the gyro's constant drift $0.01 / \mathrm{h}$, the random drift $0.002 / \mathrm{h}$, the accelerometer zero bias $100 \mathrm{u} \mathrm{g}$, the position error $20 \mathrm{~m}$, the velocity error $0.3 \mathrm{~m} / \mathrm{s}$, and the simulation time 900 s. 
The UKF filtering algorithm and the improved adaptive UKF filtering algorithm proposed in this paper are applied to the SINS/GPS integrated navigation system. The East speed error and the height error are taken as an example, and the simulation results are shown as follows. Figure 1 and Figure 2 are the eastward velocity error and height error of the system under the action of the UKF algorithm. Figure 3 and Figure 4 are the eastward speed error and high speed error of the system under the effect of improving the adaptive UKF.

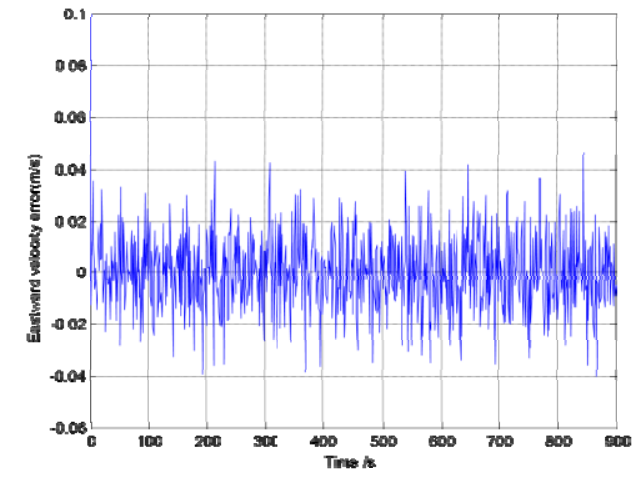

FIGURE I. EASTWARD VELOCITY ERROR (UKF ALGORITHM)

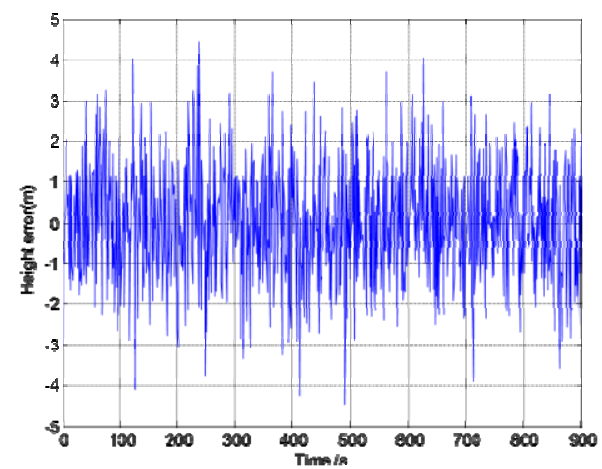

FIGURE II. HEIGHT ERROR (UKF ALGORITHM)

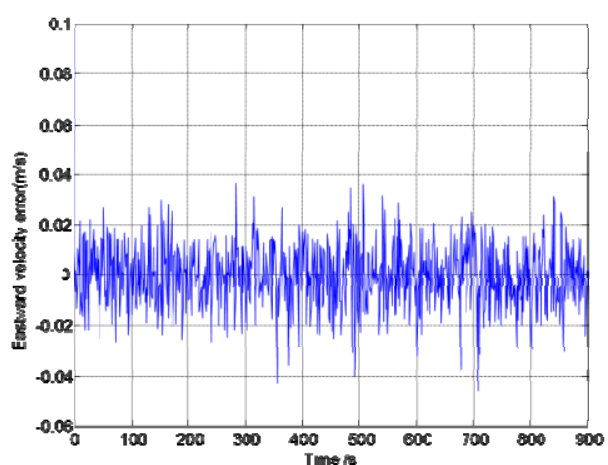

FIGURE III. EASTWARD VELOCITY ERROR (IMPROVED ADAPTIVE UKF ALGORITHM)

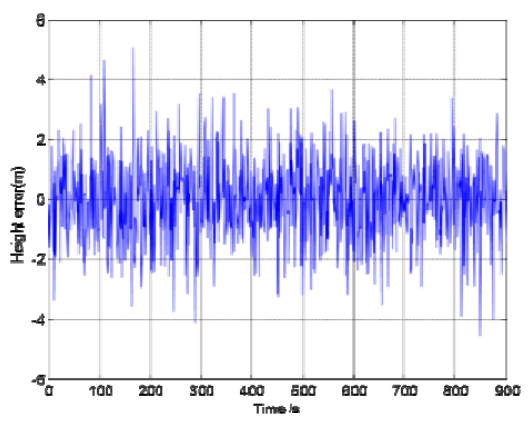

FIGURE IV. HEIGHT ERROR (IMPROVED ADAPTIVE UKF ALGORITHM)

From the simulation results, we can see that in the SINS/GPS loose integrated navigation system, the UKF filtering algorithm and the improved adaptive UKF filtering algorithm can play the role of filtering. Through comparison, it is found that the improved adaptive UKF filtering algorithm improves the performance of the filter through the introduction of adaptive factor, which makes the integrated navigation system can well restrain the influence of the initial value deviation and the fluctuation of the system state model. Therefore, the improved adaptive UKF filtering precision is better than the traditional UKF filtering algorithm.

\section{CONCLUDING REMARKS}

The improved adaptive UKF not only inherits a series of advantages of the traditional UKF filtering algorithm, but also restrates the deviation of the initial value selection of the integrated navigation system through the introduction of adaptive factors, reduces the influence of the abnormal fluctuation of the system state model on the integrated navigation system, and further improves the navigation precision of the integrated navigation system. Therefore, the improved adaptive UKF filtering algorithm applied to integrated navigation system is an effective way to improve navigation reliability and reduce error.

\section{REFERENCES}

[1] Bian Hongwei, Li An etc. Application of modern information fusion technology in integrated navigation system [M]. Beijing: National Defense Industry Press. 2010: 109-112.

[2] E. A. Wan, R. van der Merwe," The Unscented Kalman Filter for Nonlinear Estimation," in Proc. of IEEE Symposium 2000, Lake Louise, Alberta, Canada, Oct. 2000:153 158.

[3] Xu Tianlai, swam tiger, Cui Pingyuan. Research on INS/GPS integrated navigation system algorithm based on fuzzy adaptive Calman filtering [J]. Journal of Astronautics, 2005 (9): 571-575.

[4] Quan Wei, Liu Bai Qi, Gong Xiaolin, et al. Inertial / astronomical / Satellite Integrated Navigation Technology [M]. Beijing: National Defense Industry Press. 2011: 215-218.

[5] Yang Yuanxi, He Haibo, Xu Guochang. Adaptively robust filtering for kinematic geodetic positioning [J] .Journal of Geodesy, 2001, 75(2):109116.

[6] Gao Wei Guang, He Haibo, Chen Jinping. Adaptive UKF algorithm and its application in GPS/INS integrated navigation [J]. Journal of Beijing Institute of Technology, 2008(6): 505-509.

[7] Yang Yuanxi, Gao Weiguang. An optimal adaptive Kalman filter [J]. Journal of Geodesy, 2006, 80(4):177-183. 
[8] Zhang Hongqi. Application of new adaptive Kalman filtering algorithm in integrated navigation [D]. Harbin Engineering University master degree thesis, 2011,12.

[9] S. Brunke, M. Campbell. Estimation Architecture for Future Autonomous Vehicles. Proceeding of American Control Conference, 2002:1108-1114.

[10] Liu Zi Ling, Yao Zhicheng, Fan Zhiliang. Satellite / inertial integrated navigation signal simulator design [J]. radio engineering, 2014:39-42. 